Proc. Indian Acad. Sci. (Earth Planet. Sci.), Vol. 92, Number 2, July 1983, pp. 105-113.

(C) Printed in India.

\title{
Application of a semi-implicit scheme of integration to barotropic prediction
}

\author{
S S SINGH and A BANDOPADHYAY \\ Indian Institute of Tropical Meteorology. Pune 411005 , India \\ MS received 11 December 1981; revised 3 November 1982
}

\begin{abstract}
Application of a semi-implicit version of primitive equation barotropic model to predict moasoon depressions is proposed. Forecasts upto $48 \mathrm{hr}$ have been quite satisfactory. The results are compared with those obtained from an explicit version of primitive equation barotropic model developed earlier. The flow patterns from these two versions are similar with few exceptions in isolated pockets. however. the forecast movement obtained from the semi-implicit version is comparable or slightly superior to those obtained from the explicit version. The computational time in semi-implicit and explicit schemes is approximately in the ratio of $1: 2$.
\end{abstract}

Keywords. Semi-implicit scheme; barotropic model: monsoon depression.

\section{Introduction}

Systematic studies on the development of numerical weather prediction models in India were taken up nearly a decade back. Shukla and Saha (1970) used a nondivergent barotropic model with the wind at $500 \mathrm{mb}$ as input to predict the movement of a tropical cyclone in the Bay of Bengal. The explicit version (Singh and Saha 1976) of the primitive equation barotropic model were applied for the prediction of monsoon depressions and tropical., clones in tne Indian region and was applied extensively to study the impact of adunonai data collected during MONEX-79. on the barotropic prediction of monsoon depressionis by Sikka et al $(1980,1981)$.

In the present study, a semi-implicit scheme of integration to a primitive equation barotropic model has been applied to predict monsoon depressions. This scheme is similar to those by Kwizak and Robert (1971), McPherson (1971) and Halberstam (1974) in the integration of finite difference primitive equation barotropic model and the terms relevant to meteorological waves were approximated explicitly and gravitational oscillations evaluated implicitly. Thus, the restriction in time step may depend on the velocity of meteorological waves and horizontal grid resolutions, and a large time step (say $30-40 \mathrm{~min}$ ) could be used for a grid length of $100-200 \mathrm{~km}$, whereas in the explicit versions a maximum time step of $10 \mathrm{~min}$ could be used for the same grid length. The semi-implicit scheme of integration is now being used with increasing acceptance to integrate multilevel primitive equation models (Burridge 1975; Gauntlett et al 1978; McGregor et al 1978) due to considerable saving of computational time. In the study of simulation of monsoon circulation and prediction of movement of a monsoon depression. Das and Bedi (1978a, b; 1979) have applied a semi-implicit scheme of integration in a multilevel primitive equation model. The purpose of the present experiment is to extend this scheme to the multilevel primitive equation model currently being developed by our group. 
The results obtained from the semi-implicit (Scheme I) barotropic version will be compared with those obtained from the explicit version developed earlier (Singh and Saha 1976) which is hereafter referred to as Scheme II. The semi-implicit version will be described briefly in $\$ 3$.

\section{Data}

For both the versions of the model, five typical synoptic situations $(4,5$ and 6 August 1968 and 5 and 6 July 1979) dominated by monsoon depression have been selected. The free surface is assumed at $700 \mathrm{mb}$ and as such the mean height of free surface is taken $2000 \mathrm{gpm}$. The stream-line isotach for all synoptic situations at $700 \mathrm{mb}$ has been analysed manually and the wind speed and direction were picked up at 2 degree latitude-longitude intersections. A time-step of $30 \mathrm{~min}$ for the first version and $5 \mathrm{~min}$ for the second has been chosen.

\section{Method of computations}

\subsection{Model equations}

The prediction equation in Cartesian coordinate system on mercator projection are:

$$
\begin{aligned}
& \frac{\partial u}{\partial t}+m u \frac{\partial u}{\partial x}+m v \frac{\partial u}{\partial y}-f v+g m \frac{\partial h}{\partial x}=0 \\
& \frac{\partial v}{\partial t}+m u \frac{\partial v}{\partial x}+m v \frac{\partial v}{\partial y} \pm f u+g m \frac{\partial h}{\partial y}=0 \\
& \frac{\partial h}{\partial t}+m u \frac{\partial h}{\partial x}+m v \frac{\partial h}{\partial y}+h m\left(\frac{\partial u}{\partial x}+\frac{\partial v}{\partial y}\right)-h v \frac{\partial m}{\partial y}=0,
\end{aligned}
$$

where $u$ and $v$ are the component of the horizontal velocity vector ( $u$-along the $x$ coordinate pointing eastward and $v$-along the $y$-coordinate pointing northward), $h$ the height of free surface ( $h=0$ is the mean sea level), $t$ the time, $g$ the acceleration due to gravity, $m$ the map factor (secant of latitudes) and $f$ the Coriolis parameter.

\subsection{Semi-implicit scheme}

Equations (1) to (3) can be written using Leapfrog time differencing, and averaging in time the approximate linear terms:

$$
\begin{aligned}
& \bar{u}^{2 t}=u^{t-\Delta t}-\Delta t\left(m u \frac{\partial u}{\partial x}+m v \frac{\partial u}{\partial y}-f^{\prime} v\right)+f_{0} \bar{v}^{2 t} \Delta t-g m \frac{\partial \bar{h}^{2 t}}{\partial x} \cdot \Delta t, \\
& \bar{v}^{2 t}=v^{t-\Delta t}-\Delta t\left(m u \frac{\partial v}{\partial x}+m v \frac{\partial v}{\partial y}+f^{\prime} u\right)-f_{0} \bar{u}^{2 t} \Delta t-g m \frac{\partial \bar{h}^{2 t}}{\partial y} \Delta t \\
& \text { (5) }
\end{aligned}
$$




$$
\begin{aligned}
\bar{h}^{2 t}=h^{t-\Delta t}-\Delta t\left(m u \frac{\partial h}{\partial x}+m v \frac{\partial h}{\partial y}\right)-m h \Delta t \\
\\
\left(\frac{\partial \bar{u}^{2 t}}{\partial x}+\frac{\partial \bar{v}^{2 t}}{\partial y}\right)+h v \Delta t \frac{\partial m}{\partial y}
\end{aligned}
$$

where $f=f_{0}+f^{\prime}, f_{0}$ being the value of the Coriolis parameter at the centre of the domain $\left(22^{\circ} \mathrm{N}\right)$ and the time-average operator is defined as

$$
F^{2 t}=1 / 2[F(t+\Delta t)+F(t-\Delta t)]
$$

Eliminating $\vec{v}^{2 t}$ from (4) and $\bar{u}^{2 t}$ from (5), explicit equation for $\bar{u}^{2 t}$ and $\bar{v}^{2 t}$ in terms of $h^{2 t}$ can be written as

$$
\begin{aligned}
& \lambda \bar{u}^{2 t}=A-g m \Delta t\left(\frac{\partial \bar{h}^{2 t}}{\partial x}+f_{0} \Delta t \frac{\partial \bar{h}^{2 t}}{\partial y}\right) \\
& \lambda \bar{v}^{2 t}=B-g m \Delta t\left(\frac{\partial \bar{h}^{2 t}}{\partial y}-f_{0} \Delta t \frac{\partial \bar{h}^{2 t}}{\partial x}\right)
\end{aligned}
$$

where $\lambda=1+f_{0}^{2} \Delta t^{2}$ and $A$ and $B$ are given as follows:

$$
\begin{aligned}
& A=u^{t-\Delta t}+f_{0} \Delta v^{t-\Delta t}-\Delta t\left[\left(m u \frac{\partial u}{\partial x}+m v \frac{\partial u}{\partial y}-f^{\prime} v\right)+\right. \\
& \left.f_{0} \wedge\left(m u \frac{\partial v}{\partial x}+m v \frac{\partial v}{\partial y}+f^{\prime} u\right)\right] \\
& B=v^{t-\Delta t}-f_{0} \Delta t u^{t-\Delta t}+\Delta t\left[f_{0} \Delta t\left(m u \frac{\partial u}{\partial x}+m v \frac{\partial u}{\partial y}-f^{\prime} v\right)-\right. \\
& \left.\left(m u \frac{\partial v}{\partial x}+m v \frac{\partial v}{\partial y}+f^{\prime} u\right)\right]
\end{aligned}
$$

Finally differentiating (7) with respect to $x$ and (8) with respect to $y$ (neglecting the latitudinal variation of $m$ ) and substituting the velocity divergence into (6) the continuity equation yields:

$$
\nabla^{2} h^{2 t}-\lambda \bar{h}^{2 t}\left(\Delta t^{2} g h\right)^{-1}=\lambda Q^{N}\left(\Delta t^{2} g h\right)^{-1}
$$


where

$$
\begin{aligned}
Q^{N}= & \frac{h \Delta t}{\lambda}\left(m \frac{\partial A}{\partial x}+m \frac{\partial B}{\partial y}\right)+ \\
& \Delta t\left(m u \frac{\partial h}{\partial x}+m v \frac{\partial h}{\partial y}\right)-\Delta t h v \frac{\partial m}{\partial y}-h^{t-\Delta t}
\end{aligned}
$$

Equation (9) can be solved for $\bar{h}^{2 t}$ numerically by the method of relaxation with appropriate boundary condition, whilst $\bar{u}_{i}^{2 t}$ and $\bar{v}^{2 t}$ may be obtained from (7) and (8) respectively. Finally the values of all primary variables at $t+\Delta t$ are obtained by using relations :

$$
\begin{aligned}
& u^{i+\Delta t}=2 \bar{u}^{2 t}-u^{t-\Delta t}, \\
& v^{t+\Delta t}=2 \bar{v}^{2 t}-v^{t-\Delta t}, \\
& h^{t+\Delta t}=2 \bar{h}^{2 t}-h^{t-\Delta t}
\end{aligned}
$$

\subsection{Difference scheme, initialisation and boundary conditions}

The space difference scheme is adapted from McPherson (1971). The difference equations are formulated on an offset grid lattice; in other words the geopotential height field is defined on all grid points and " and $v$ components a re defined at the centre of the squares.

For obtaining the initial balance between mass and wind fields, the nonlinear balance equation is solved for geopotential height with appropriate boundary conditions using non-divergent wind as basic input.

Cyclic continuity in the east-west and no flow across the north-south boundaries arc specified as the lateral boundary conditions. A detailed description of initialization and boundary conditions has been given by Singh et al (1980).

\section{Comparison of for ecast results}

Since the results of the initialization and forecast experiments are similar for all the synoptic situations, the figures for 4 August only will be presented and wherever necessary results of other synoptic situations will be discussed. Figure 1 shows the initial stream function chart (00 GMT $700 \mathrm{mb} 4$ August 1968) and figure 2 presents the $24 \mathrm{hr}$ forecast from both versions together with the verification chart (00 GMT $700 \mathrm{mb} 5$ August 1968). The $48 \mathrm{hr}$ forecast and corresponding verification (6 August 1968) charts are shown in figure 3 . Tables $I$ and 2 present the positional error of the iorecast from Schemes I and II respectively for the input of 4, 5 and 6 August 1968 and 5 and 6 July 1979. The verification statistics computed separately for $u$ - and $v$-fields are also presented in tabies 1 and 2 . It gives the root mean square errors and correlation coefficients between actual and forecast for the whole forecast domain. It may be seen that on average (of five cases) the forecast movement appears to be slightly better in the semi-implicit version than those obtained from the explicit version. The verification statistics also reflects the above conclusion i.e., the prediction of $u$-and $v$ fields by semi-implicit version is slightly better or comparable than those obtained 


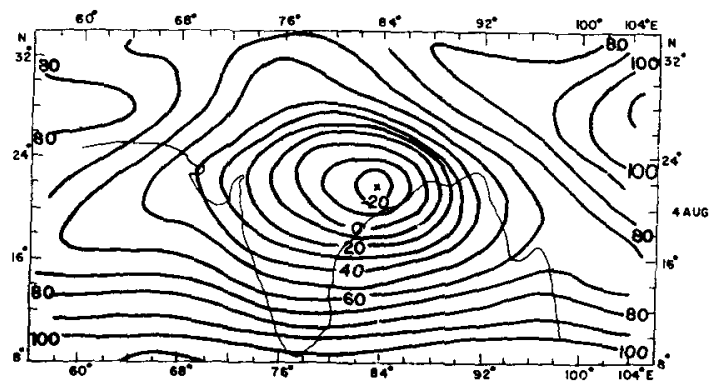

Figure 1. Initial stream function chart (00 GMT, $700 \mathrm{mb} 4$ August 1968).
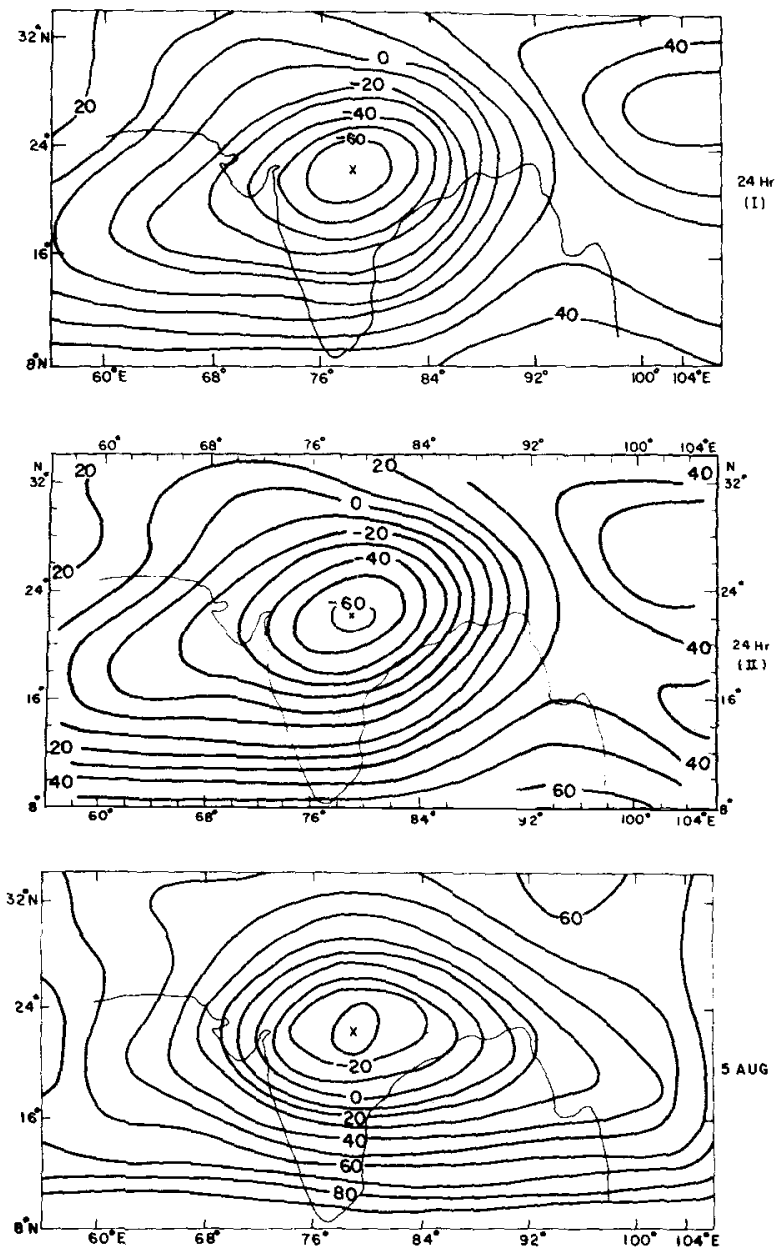

Figure 2. $24 \mathrm{hr}$ forecast stream function charts from first and second versions of the model and corresponding verification chart (00 GMT. $700 \mathrm{mb} 5$ August 1968). 

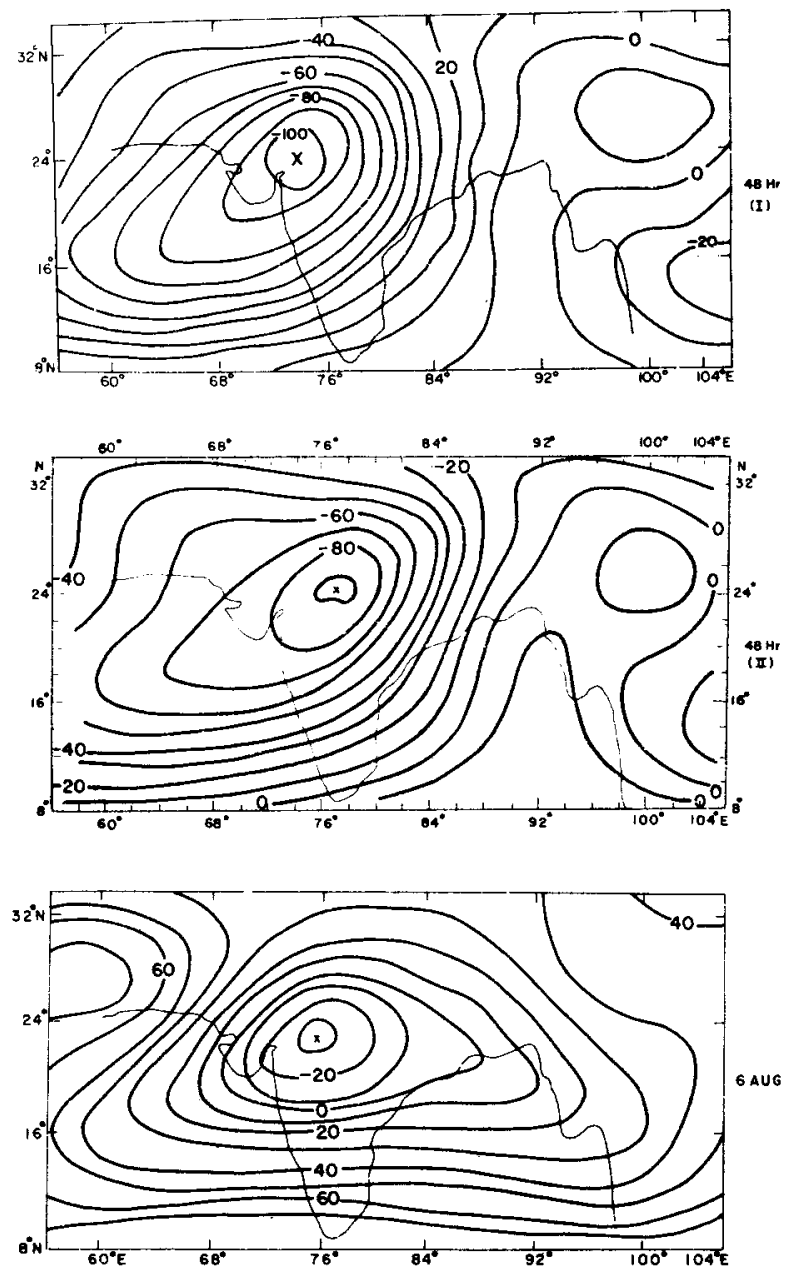

Figure 3. Same as figure 2 except for $48 \mathrm{hr}$ forecast charts and corresponding verification chart (00 GMT, $700 \mathrm{mb} 6$ August 1968).

from the explicit version. The computational time required by two schemes is in the ratio of $1: 2$. As expected, the semi-implicit scheme reduces the computational requirement considerably.

It may be pointed out that both the versions not only differ in time integration schemes but there are a number of other differences in the formulations. The semiimplicit model is formulated on offset grid rather than the regular grid as is the case with the second version and as such the space derivatives a re approximated differently in two models. Thus, the difference in the forecast is not due to different integration schemes alone but due to other factors as well. 


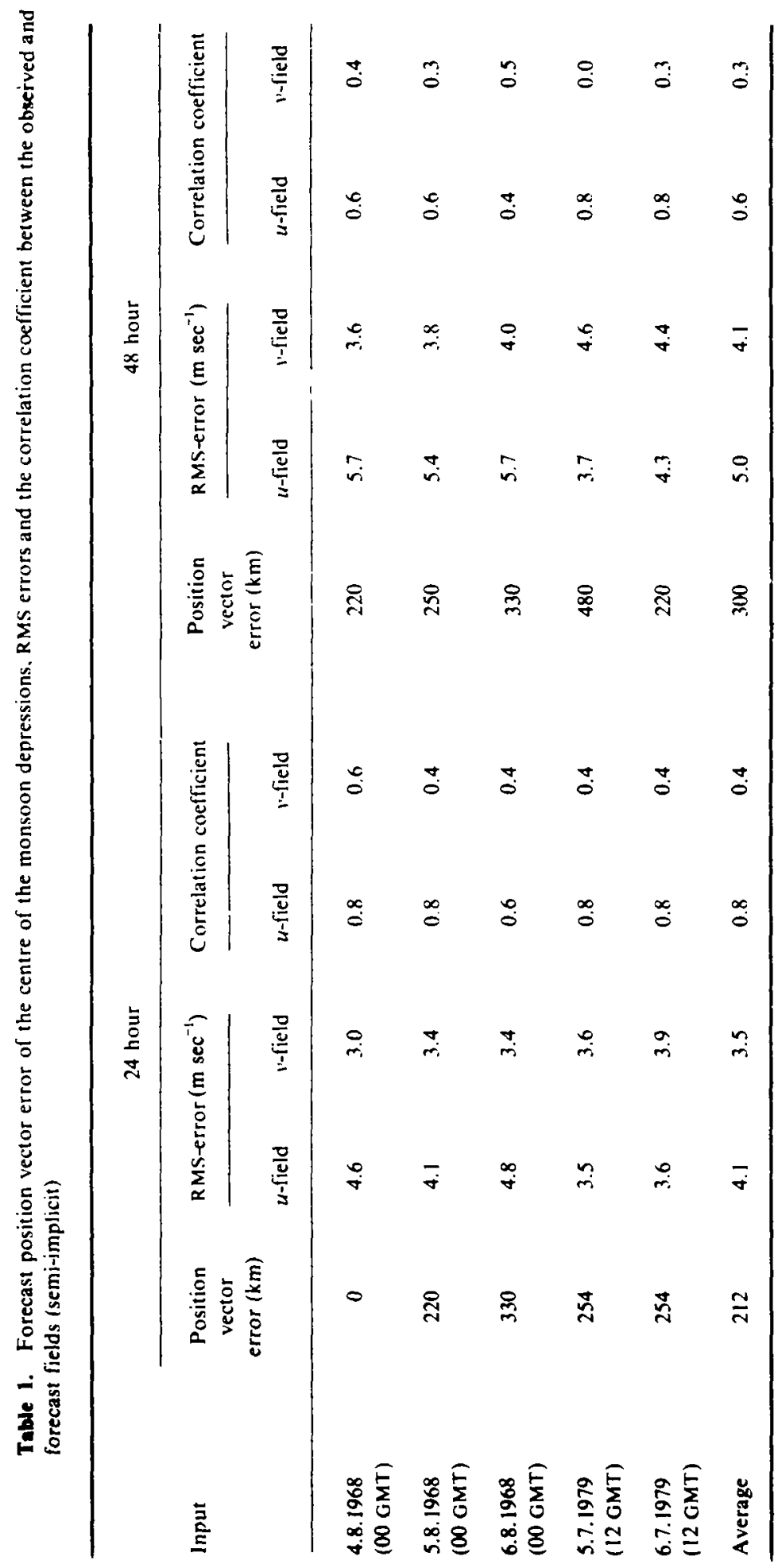




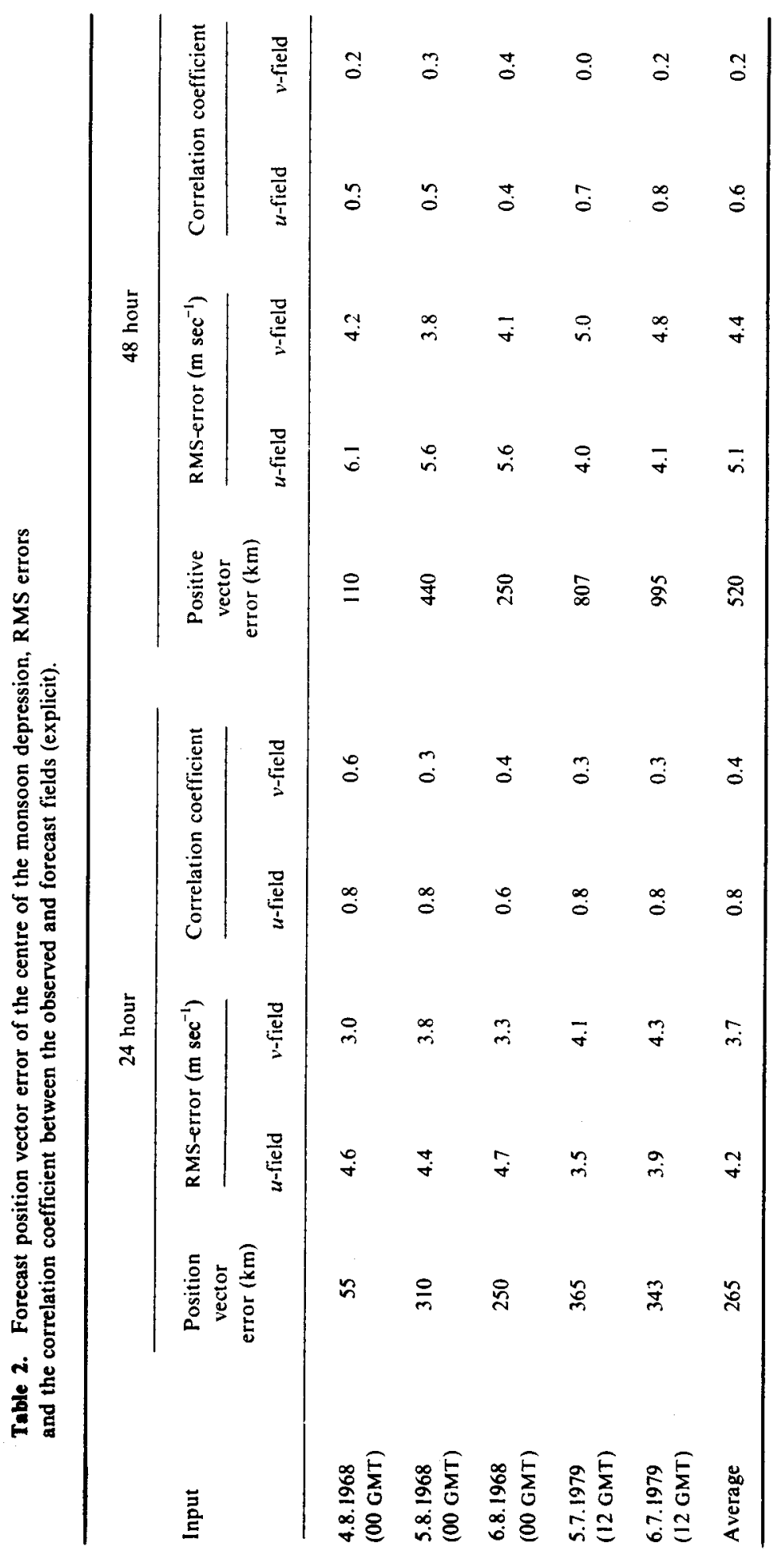




\section{Conclusions}

A semi-implicit scheme of integration has been successfully applied to predict monsoon depressions. The results are comparable or slightly superior to those obtained from the explicit version. The computational time as expected is least in this version as compared to the explicit version. A successful application of the semi-implicit analogue in the present study has encouraged the authors to apply this scheme to the multilevel primitive equation model currently under development.

\section{Acknowledgements}

Authors thank the Director, Indian Institute of Tropical Meteorology and Dr D R Sikka for their interest in this study.

\section{References}

Burridge D M 1975 Q. J. R. Meteorol. Soc. 101777

Das P K and Bedi H S 1978a Indian J. Meteorol. Geophys. 29375

Das P K and Bedi H S 1978b Monsoon dynamics (Cambridge: University Press) p. 351

Das P K and Bedi H S 1979 Proc. Indian Acad. Sci. (Engg. Sci.) 217

Gauntlett D J, Leslie L M and McGregor J L 1978 Q. J. R. Meteorol. Soc. 104103

Halberstam I 1974 J. Atmos. Sci. 311964

Kwizak M and Robert A J 1971 Mon. Weath. Rev. 9932

McGregor J L, Leslie L M and Gauntlett D J 1978 Mon. Weath. Rev. 106427

McPherson R D 1971 Mon. Weath. Rev. 99242

Shukla J and Saha K R 1970 J. Meteorol. Soc. Jpn. 48405

Sikka D R, Singh S S and Rajamani S 1980 Results of the summer MONEX Field Phase Research, Part A, FGGE Operations Report, 9151

Sikka D R, Sınha S, Singh S S, Kulkarni P L and Kulkarni A A 1981 Early results of FGGE and monsoon experiments, Proc. Int. Conf., Tallahassee, USA

Singh S S and Saha K R 1976 J. Appl. Meteorol. 15805

Singh S S, Kulkarni A A and Sikka D R 1980 Mon. Weath. Rev, 1081315 